05,11

\title{
Особенности критической динамики в магнитноодноосном гексаферрите $\mathrm{PbFe}_{12} \mathrm{O}_{19}$
}

\author{
() А.А. Безлепкин, С.П. Кунцевич \\ Харьковский национальный университет им. В.Н. Каразина, \\ Харьков, Украина \\ E-mail: anatoliy.a.bezlyepkin@univer.kharkov.ua
}

(Поступила в Редакцию 24 апреля 2017 г.)

\begin{abstract}
Сформулированы условия, при которых термодинамическая теория критического замедления скорости релаксации параметра порядка описывает поведение магнитноодноосных кристаллов. С учетом сформулированных условий изучены особенности динамической магнитной восприимчивости и критического замедления скорости релаксации намагниченности $\mathrm{PbFe}_{12} \mathrm{O}_{19}$ в области температуры Кюри. Полученные экспериментальные результаты хорошо согласуются с капельной моделью фазового перехода. В рамках капельной модели на основе экспериментальных данных получено оценочное значение корреляционной длины для намагниченности в температурной области критического замедления скорости релаксации.
\end{abstract}

DOI: 10.21883/FTT.2018.01.45289.139

\section{1. Введение}

В работе [1] была создана термодинамическая теория критического замедления скорости релаксации параметра порядка. Теория основывалась на феноменологическом предположении, что критическая динамика носит чисто релаксационный характер. Считалось, что в выражении для скорости изменения параметра порядка кинетический коэффициент остается постоянным. Было показано, что в этом случае переход параметра порядка к равновесному значению происходит очень медленно, а время релаксации $\tau$ при приближении к температуре фазового перехода $T_{c}$ со стороны более низких температур растет в соответствии с выражением [1]

$$
\tau \sim\left(T_{c}-T\right)^{-1} .
$$

В магнитоупорядоченных веществах, если не создавать специальных условий, релаксационные процессы в магнитном поле будут происходить при наличии прецессионного движения магнитных моментов, что не учитывается в термодинамической теории. В магнитноодноосном кристалле существуют области однородной намагниченности - домены с ориентацией вектора намагниченности вдоль поля анизотропии - и переходные области между доменами - доменные границы. Ширина доменных границ обусловлена конкуренцией между ростом энергии анизотропии и уменьшением обменной энергии при увеличении ширины границы. В высокоанизотропных магнитноодноосных кристаллах ширина доменных границ гораздо меньше ширины доменов, а отношение объемов доменов к объему доменных границ составляет $\sim 1 \cdot 10^{3}[2]$. Поэтому при намагничивании таких кристаллов слабым магнитным полем, приложенным вдоль поля магнитной кристаллографической анизотропии, влияние доменных границ носит косвенный характер, их размеры практически не изменяются, а основной вклад в намагниченность вносят домены, в которых векторы намагниченности ориентированы вдоль переменного магнитного поля. В этом случае в уравнении движения для намагниченности отсутствует прецессионное слагаемое, а динамика намагниченности носит релаксационный характер.

В работе [3] была рассмотрена динамика намагниченности в магнитном одноосном кристалле при ориентации вектора намагниченности и вектора магнитного поля вдоль поля магнитной анизотропии. В этом случае действительная $\chi^{\prime}$ и мнимая $\chi^{\prime \prime}$ составляющие магнитной восприимчивости имеют вид $[3,4]$

$$
\chi^{\prime}=\frac{\chi_{0}}{1+\omega^{2} \tau^{2}}, \quad \chi^{\prime \prime}=\frac{\chi_{0} \omega \tau}{1+\omega^{2} \tau^{2}},
$$

где $\chi_{0}-$ величина магнитной восприимчивости при $\omega \rightarrow 0 ; \omega-$ частота внешнего переменного магнитного поля; $\tau$ - время релаксации при отклике намагниченности на переменное магнитное поле.

Из соотношений $(1),(2)$ следует, что если $\chi_{0}=$ const при критическом замедлении скорости релаксации $\tau \rightarrow \infty, \chi^{\prime} \rightarrow 0$, а $\chi^{\prime \prime} \rightarrow \chi_{0} / 2$ при $\omega \tau=1$.

При произвольной ориентации образца относительно магнитного поля $\chi_{0}$ будет зависеть от температуры и магнитного поля. Для магнитноодноосных кристаллов при ориентации магнитного поля вдоль поля магнитной анизотропии и соответственно вдоль направления легкого намагничивания величина $\chi_{0}$ постоянна и определяется выражением [5]

$$
\chi_{0}=N^{-1}=\text { const, }
$$

где $N$ - размагничивающий фактор образца.

Соотношение (3) выполняется в интервале магнитных полей, задаваемом выражением [5]

$$
H<N I_{s}
$$

где $I_{s}$ - намагниченность насыщения [6]. 
Величина $I_{s}$ мала в области температуры Кюри, поэтому условие $\chi_{0}=$ const при фазовом переходе будет выполняться в малых магнитных полях. Для наблюдения критического замедления скорости релаксации при изучении $\chi^{\prime}$ и $\chi^{\prime \prime}$ необходимо выполнение условий $(3),(4)$ при ориентации переменного магнитного поля вдоль поля магнитной анизотропии в магнитноодноосном кристалле.

В настоящей работе с учетом сформулированных условий изучены особенности критической динамики в гексаферрите $\mathrm{PbFe}_{12} \mathrm{O}_{19}(\mathrm{PbM})$ в области температуры Кюри. Исходя из экспериментальных данных, получено оценочное значение корреляционной длины для намагниченности в температурной области критического замедления скорости релаксации.

\section{2. Образцы и методика измерений}

Монокристаллы РbM были выращены методом раствора в расплаве флюса $\mathrm{PbO}$. Синтез производился в интервале температур $950-1050^{\circ} \mathrm{C}$ на вращающейся затравке. Фазовый анализ контролировался рентгенографическим методом. Постоянные кристаллической решетки исследуемого феррита при комнатной температуре: $a=0.589 \mathrm{~nm}, c=2.312 \mathrm{~nm}$.

Действительная часть динамической магнитной восприимчивости $\chi^{\prime}$ определялась резонансным методом, исходя из экспериментальных значений резонансной частоты $L C$-контура, в катушке индуктивности которого помещался исследуемый образец. Образцы имели форму, близкую к сферической, с диаметром $\sim 5 \mathrm{~mm}$. Для определения $\chi^{\prime}$ использовалась формула

$$
\mu^{\prime}(t) \cong 1+\chi^{\prime}(T)=B\left[f_{1}^{-2}(T)-f_{2}^{-2}(T)\right],
$$

где $\mu^{\prime}(T)$ - действительная часть магнитной проницаемости; $B$ - величина, зависящая от параметров контура и включающая постоянные численные коэффициенты, в том числе и коэффициент заполнения катушки образцом; $f_{1}(T)$ - частота контура с образцом при температуре $T ; f_{2}(T)$ - частота пустого контура при температуре $T$.

Поскольку определить точное значение коэффициента заполнения катушки образцом не представляется возможным, величина $\chi^{\prime}$ приведена в относительных единицах. Для измерения характеристик контура использовался анализатор спектров СК4-59. Точность определения частоты контура $\sim 2 \mathrm{kHz}$.

Катушка индуктивности изготавливалась из платиновой проволоки, помещалась в электрическую печь, питание которой осуществлялось постоянным током. Нагреватель наматывался бифилярно на медную трубку, которая обеспечивала однородность температуры в рабочем пространстве печи. Исследования были проведены в переменных магнитных полях, не превышающих $1 \cdot 10^{-3}$ Oe.

\section{3. Экспериментальные результаты и обсуждение}

На рис. 1 (кривая 1) представлена зависимость резонансной частоты $L C$-контура с кристаллом $\mathrm{PbFe}_{12} \mathrm{O}_{19}$, ориентированным гексагональной осью с вдоль переменного магнитного поля с частотой $63.8 \mathrm{MHz}$ в интервале

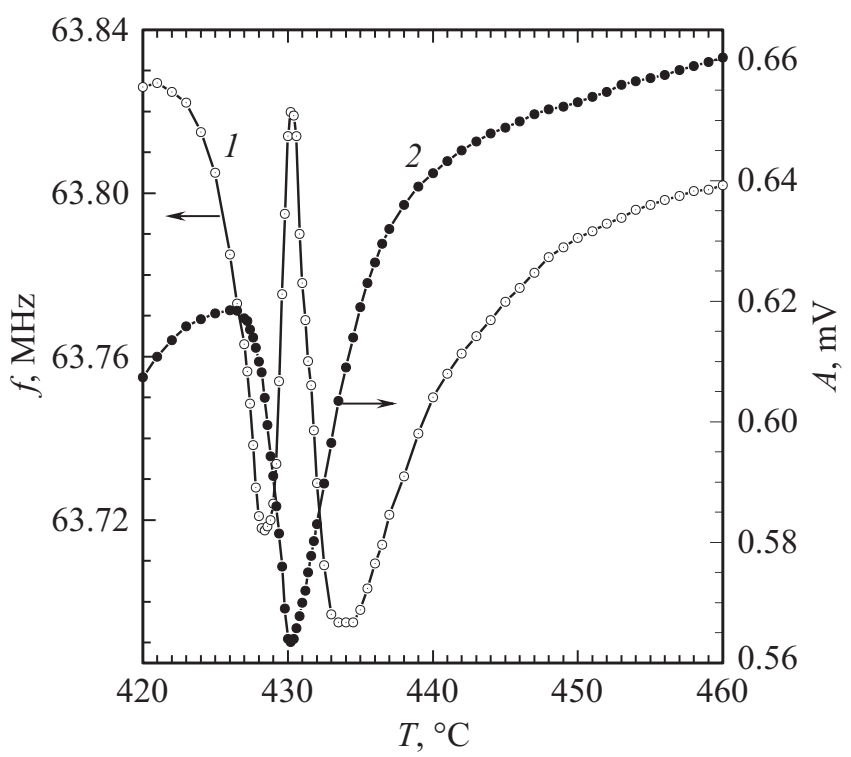

Рис. 1. Температурная зависимость частоты измерительного контура (1) и напряжения на контуре (2) с образцом $\mathrm{PbFe}_{12} \mathrm{O}_{19}$, ориентированным осью с вдоль переменного магнитного поля катушки контура.

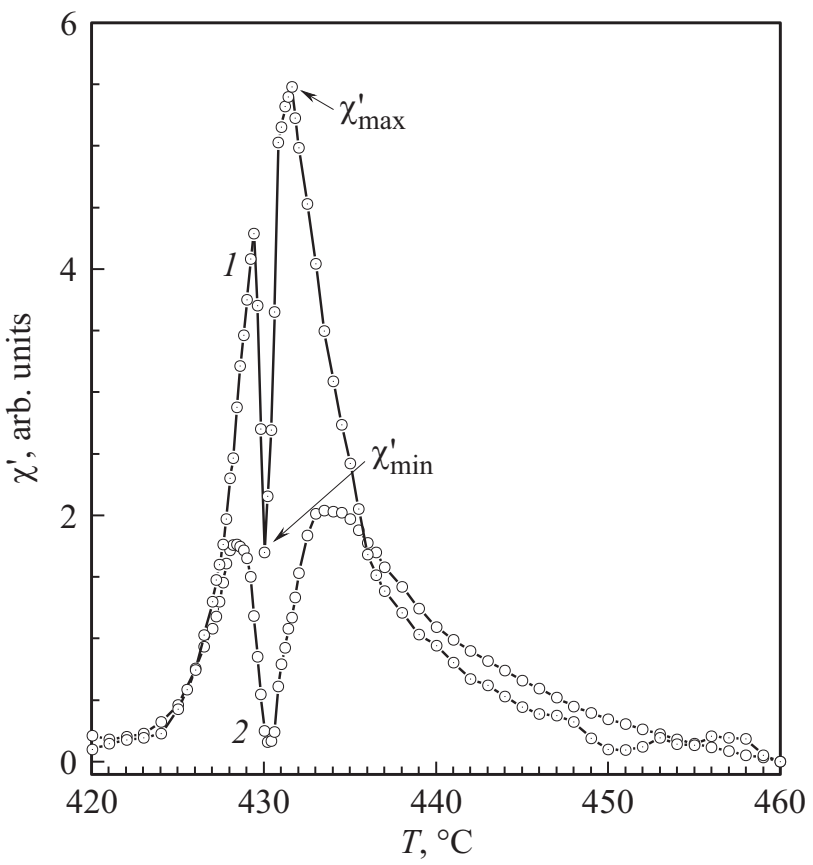

Рис. 2. Температурная зависимость действительной части магнитной восприимчивости $\chi^{\prime}$ образца $\mathrm{PbFe}_{12} \mathrm{O}_{19}$ в направлении легкого намагничивания вблизи частот $24.3 \mathrm{MHz}(1)$ и $63.8 \mathrm{MHz}$ (2) в области температуры Кюри. 
температур $420-460^{\circ} \mathrm{C}$. Определенные из экспериментальных данных с помощью соотношения (5) величины $\chi^{\prime}$ в относительных единицах, приведены на рис. 2 (кривая 2). Как видно из рисунка, на фоне широкого максимума $\chi^{\prime}$ в области температуры $430^{\circ} \mathrm{C}$ имеет место пикообразное уменьшение $\chi^{\prime}$. Минимальное значение $\chi^{\prime}$ имеет при температуре $T_{c}$. Из соотношения (2) следует, что при критическом замедлении скорости релаксации и росте $\tau$ уменьшение $\chi^{\prime}$ должно сопровождаться ростом $\chi^{\prime \prime}$. Величина $\chi^{\prime \prime}$ обратно пропорциональна добротности контура и обратно пропорциональна напряжению на нем. На рис. 1 (кривая 2) представлена зависимость напряжения на $L C$-контуре в интервале температур $420-460^{\circ} \mathrm{C}$. Как видно из рисунка, при температуре минимума $\chi^{\prime}$ величина напряжения минимальна, что соответствует максимальному значению $\chi^{\prime \prime}$. Поэтому пикообразное уменьшение $\chi^{\prime}$ обусловлено ростом $\tau$ и соответственно критическим замедлением скорости релаксации параметра порядка - намагниченности. В работе [7] показано, что температура Кюри, определенная по максимуму $\chi^{\prime \prime}$, совпадает с температурой Кюри, определенной нейтронографическим методом. Поэтому температура $T_{c}=430^{\circ} \mathrm{C}$, соответствующая максимуму $\chi^{\prime \prime}$, является температурой Кюри.

Рассматриваемые особенности поведения $\chi^{\prime}$ не наблюдались на кристаллах, не обладающих осью легкого намагничивания. На рис. 3 представлена зависимость $\chi^{\prime}(T)$ гексаферрита $\mathrm{Zn}_{2} \mathrm{Ba}_{2} \mathrm{Fe}_{12} \mathrm{O}_{22}\left(\mathrm{Zn}_{2} Y\right)$, имеющего плос-

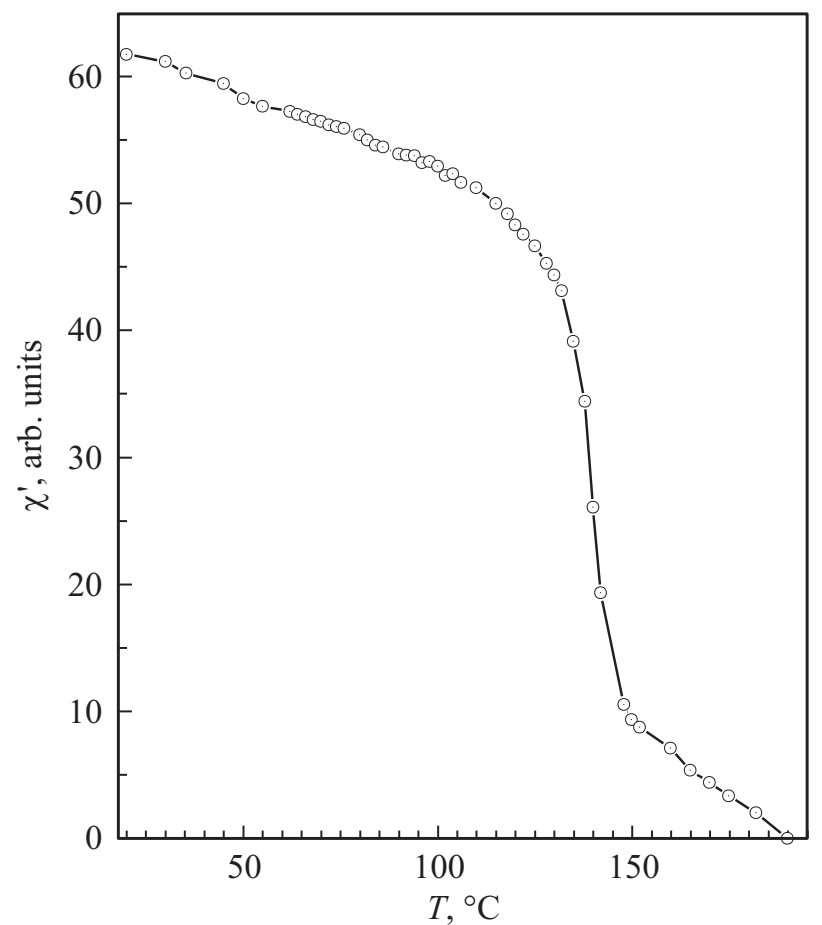

Рис. 3. Температурная зависимость действительной части магнитной восприимчивости $\chi^{\prime}$ образца $\mathrm{Zn}_{2} \mathrm{Ba}_{2} \mathrm{Fe}_{12} \mathrm{O}_{22}$ $\left(\mathrm{Zn}_{2} Y\right)$ в направлении легкого намагничивания вблизи частоты $41.5 \mathrm{MHz}$ в области температуры Кюри.

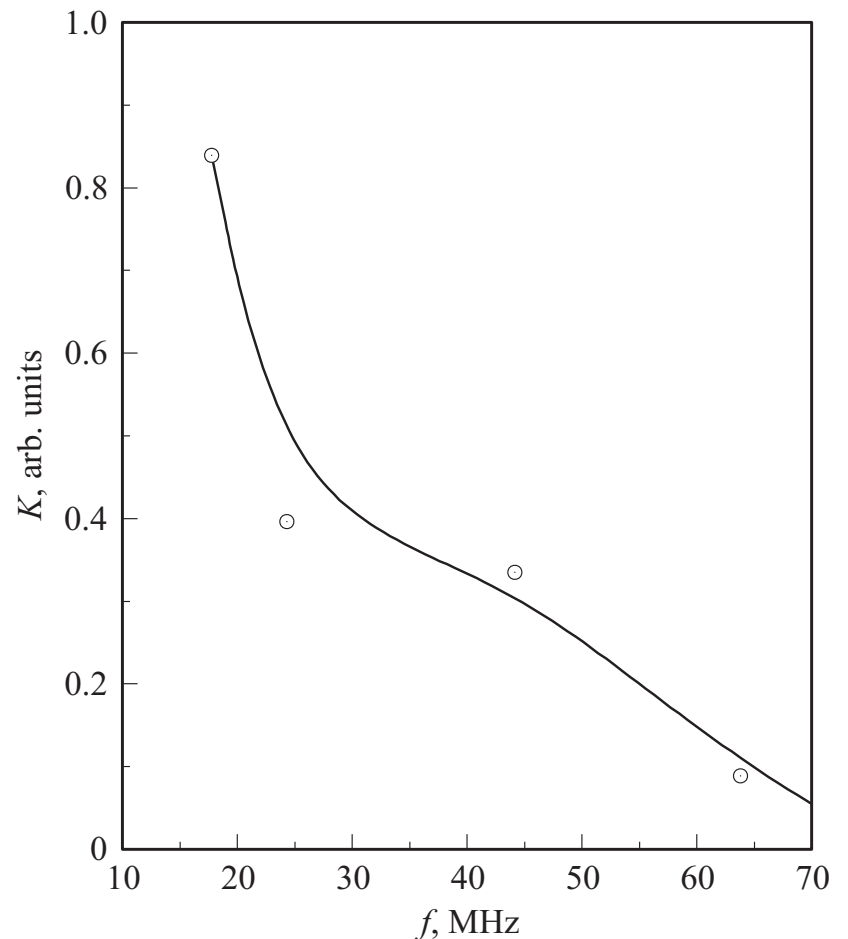

Рис. 4. Зависимость параметра $K$ от частоты для феррита $\mathrm{PbFe}_{12} \mathrm{O}_{19}$.

кость легкого намагничивания [4]. Переменное магнитное поле ориентировано в базисной плоскости кристалла, которая является плоскостью легкого намагничивания. Температура Кюри феррита $\operatorname{Zn}_{2} Y T_{c}=140^{\circ} \mathrm{C}$ [4]. Как видно из рисунка, в области температуры Кюри не наблюдается пикообразного уменьшения $\chi^{\prime}$.

На рис. 2 (кривая 1) представлена зависимость $\chi^{\prime}(T)$ при частоте переменного поля $24.3 \mathrm{MHz}$ в интервале температур $420-460^{\circ} \mathrm{C}$. Кристалл $\mathrm{Pb} M$ ориентировался гексагональной осью вдоль переменного магнитного поля. Как видно из рисунка, относительная величина пика при температуре Кюри несколько меньше пика на частоте $63.8 \mathrm{MHz}$. Для характеризации их соотношения использовалась величина $K$, равная

$$
K=\frac{\chi_{\min }^{\prime}}{\chi_{\max }^{\prime}},
$$

где $\chi_{\min }^{\prime}$ - минимальное значение, а $\chi_{\max }^{\prime}$ - максимальное значение в окрестности точки Кюри.

На рис. 4 представлена зависимость $K$ от частоты переменного магнитного поля. При уменьшении частоты растет $K$. При $K=1$ пик не наблюдается. Величина $K=1$ соответствует частоте $f_{\min } \approx 1 \cdot 10^{7} \mathrm{~Hz}$.

Полученные экспериментальные результаты указывают на следующий физический механизм, ответственный за наблюдаемые особенности критической динамики, согласно капельной модели фазового перехода из магнитоупорядоченного состояния в парамагнитное [8]. В области критической динамики весь объем кристалла 
заполнен каплями, представляющими собой флуктуационные магнитоупорядоченные корреляционные образования, характеризуемые определенным значением параметра порядка - намагниченности. В течение времени жизни корреляционных образований могут возбуждаться колебательные моды намагниченности. Пространственные колебания намагниченности вследствие магнитоупругой связи будут возбуждать упругие колебания кристаллической решетки с длиной волны

$$
\lambda=v \cdot f^{-1},
$$

где $v$ - скорость упругой волны в кристалле.

Феррит содержит капли всех размеров, вплоть до максимального, равного корреляционной длине $\xi$ [8]. В кристалле нет капель размера $R>\xi$, но есть капли с размерами $R<\xi$. Критическая динамика, согласно [9], возникает при выполнении условия

$$
\frac{2 \pi}{\lambda} \xi=\xi q>1
$$

где $q$ - модуль волнового вектора.

Переход от критической динамики к гидродинамическому режиму соответствует выполнению условия [9]

$$
\frac{2 \pi}{\lambda} \xi<1
$$

Поэтому предельная частота $f_{\text {lim }} \approx 10 \mathrm{MHz}$ соответствует предельной длине волны $\lambda_{\text {lim }}$, больше которой критическая динамика отсутствует. Из соотношений (8), (9) следует:

$$
\xi \approx \frac{\lambda_{\lim }}{2 \pi}=\frac{V}{2 \pi f_{\lim }} .
$$

При увеличении частоты уменьшается длина волны, в рассматриваемый процесс вовлекаются флуктуационные образования с $R<\xi$, что приводит к росту объема кристалла, охваченного критической динамикой и соответственно к уменьшению $\chi^{\prime}$ в области температуры Кюри.

В литературе отсутствуют сведения о скорости распространения упругих волн в $\mathrm{PbFe}_{12} \mathrm{O}_{19}$. Поэтому для оценки величины $\xi$ использовалось значение скорости продольных упругих волн при комнатной температуре $v=7.1 \cdot 10^{3} \mathrm{~m} / \mathrm{s}$ для феррита железо-иттриевого граната $\mathrm{Y}_{3} \mathrm{Fe}_{5} \mathrm{O}_{12}$, имеющего близкий элементный состав и родственный тип химических связей $[10]$. В работах $[11,12]$ показано, что изменение величины $v$ в феррите $\mathrm{Y}_{3} \mathrm{Fe}_{5} \mathrm{O}_{12}$ в области температуры Кюри и в интервале температур $20-450^{\circ} \mathrm{C}$ не превышает $5 \%$.

Воспользовавшись значением $v=7.1 \cdot 10^{3} \mathrm{~m} / \mathrm{s}$ и величиной $f_{\lim } \approx 10 \mathrm{MHz}$ получим приближенное оценочное значение $\xi \approx 110 \mu \mathrm{m}$.

\section{4. Выводы}

1. Термодинамическая теория критического замедления при выполнении определенных условий описывает поведение магнитноодноосных кристаллов $\mathrm{PbFe}_{12} \mathrm{O}_{19}$ в области фазового перехода.

2. Полученные экспериментальные результаты хорошо согласуются с капельной моделью фазового перехода из магнитоупорядоченного состояния в парамагнитное.

3. Использование капельной модели позволяет на основе экспериментальных данных рассчитать корреляционную длину в области критического замедления скорости релаксации параметра порядка.

\section{Список литературы}

[1] Л.Д. Ландау, Н.М. Халатников. Докл. АН СССР, 96, 469 (1954).

[2] С.В. Вонсовский. Магнетизм. Наука, М. (1970). 1032 с.

[3] А.А. Безлепкин, С.П. Кунцевич. Весн. ХНУ. Сер. физ. 1135, 21,71 (2014).

[4] Х. Смит, Я. Вейн. Ферриты. ИЛ, М. (1962). 540 с.

[5] Г.С. Кринчик. Физика магнитных явлений. МГУ, М. (1976). $367 \mathrm{c}$.

[6] А.А. Безлепкин, С.П. Кунцевич, С.П. Палехин. Изв. вузов МВ и ССО СССР. Физика 7, 111 (1987).

[7] Г.М. Драбкин, Я.А. Косман, В.В. Рудов. Письма ЖЭТФ 15, 379 (1972).

[8] Л.П. Каданов. Критические явления, гипотеза универсальности, скейлинг и капельная модель. В сб. Квантовая теория поля и физика фазовых переходов. Мир, М. (1975). C. 7-32.

[9] E. Frey, F. Schwabl. Adv. Phys. 43, 5, 577 (1994).

[10] A.E. Klark, R.E. Strakha. J. Appl. Phys., 32, 6, 1172 (1961).

[11] И.К. Камилов, Х.К. Алиев. ЖЭТФ 65, 5, 11, 1911 (1973).

[12] Ю.А. Буренков, С.П. Никаноров. ФТТ 44, 2, 307 (2002). 